\title{
ESTIMATING GROSS PRIMARY PRODUCTION IN CROPLAND WITH HIGH SPATIAL AND TEMPORAL SCALE REMOTE SENSING DATA
}

\author{
Shangrong $\operatorname{Lin}^{1,2}$, Jing $\operatorname{Li}^{2, *}$, Qinhuo Liu ${ }^{1,2,3, *}$ \\ 1 College of Resources and Environment, University of Chinese Academy of Sciences, Beijing 100049, China \\ 2 State Key Laboratory of Remote Sensing Science, Institute of Remote Sensing and Digital Earth, Chinese Academy of Sciences, \\ Beijing 100101, China ; shangrong_edu@ outlook.com \\ 3 Joint Center for Global Change Studies (JCGCS), Beijing 100875, China;
}

\section{Commission III, WG III/10}

KEY WORDS: Cropland, GOES-13 geostationary, Gross Primary Production, Landsat NDVI, MODIS NDVI/EVI product, carbon cycle, Photosynthesis active radiation (PAR).

\begin{abstract}
:
Satellite remote sensing data provide spatially continuous and temporally repetitive observations of land surfaces, and they have become increasingly important for monitoring large region of vegetation photosynthetic dynamic. But remote sensing data have their limitation on spatial and temporal scale, for example, higher spatial resolution data as Landsat data have 30-m spatial resolution but 16 days revisit period, while high temporal scale data such as geostationary data have 30-minute imaging period, which has lower spatial resolution $(>1 \mathrm{~km})$. The objective of this study is to investigate whether combining high spatial and temporal resolution remote sensing data can improve the gross primary production (GPP) estimation accuracy in cropland. For this analysis we used three years (from 2010 to 2012) Landsat based NDVI data, MOD13 vegetation index product and Geostationary Operational Environmental Satellite (GOES) geostationary data as input parameters to estimate GPP in a small region cropland of Nebraska, US. Then we validated the remote sensing based GPP with the in-situ measurement carbon flux data. Results showed that: 1) the overall correlation between GOES visible band and in-situ measurement photosynthesis active radiation (PAR) is about $50 \%\left(\mathrm{R}^{2}=0.52\right)$ and the European Center for Medium-Range Weather Forecasts ERA-Interim reanalysis data can explain $64 \%$ of PAR variance $\left(\mathrm{R}^{2}=0.64\right)$; 2) estimating GPP with Landsat 30-m spatial resolution data and ERA daily meteorology data has the highest accuracy $\left(\mathrm{R}^{2}=0.85\right.$, RMSE < $3 \mathrm{gC} / \mathrm{m} 2 /$ day), which has better performance than using MODIS 1-km NDVI/EVI product import; 3) using daily meteorology data as input for GPP estimation in high spatial resolution data would have higher relevance than 8-day and 16-day input. Generally speaking, using the high spatial resolution and high frequency satellite based remote sensing data can improve GPP estimation accuracy in cropland.
\end{abstract}

\section{INTRODUCTION}

Gross Primary Production (GPP) is one of the most important components of for terrestrial ecosystem carbon cycle and global change monitoring. Currently, Light-use-efficiency (LUE) models would be one of the most effective model which have sample data input but higher estimation precisely in different scale. Monteith (1972) first raised the framework of LUE models to estimated GPP:

$G P P=L U E \times A P A R=L U E \max \times f($ stress $) \times F P A R \times P A R \cdot(1)$

In equation (1), APAR is the absorbed photosynthesis active radiation, calculated by the fraction of absorbed active radiation (FPAR) with the photosynthesis active radiation. LUEmax is the potential LUE under no environmental stress, but mostly the LUE stresses by many factors such as temperature, water content, and light quality (Kalfas et al, 2011; Wu et al, 2008; Maselli et al.2009; Suyker and Verma 2012; Nguy-Robertson et al, 2015).Since satellites can supply large scale observation of terrestrial vegetation, a diverse set of satellite based models can apply to model GPP from small region to whole continent (Field et al, 1995; Running et al, 2000; Xiao et al, 2004, Yuan et al, 2007, King et al, 2011, Mahadevan et al, 2008). Different models would have different parameterization to the stress factor and it can be applied in different temporal and spatial resolution.

Nowadays, most of the LUE based models needs the satellite and reanalysis meteorology data input, but these data are mostly at lower spatial resolution. For instance, reanalysis climate data such as European Center for Medium-Range Weather Forecasts (ECMWF) ERA-Interim reanalysis (ERA-Interim), or North America Regional Reanalysis (NARR), Modern Era Retrospective Analysis for Research and Applications(MERRA) data would have high temporal resolution (daily) but rather coarse spatial resolution (> 0.5 degree). However, since some MODIS data based remote sensing based-GPP model have a higher resolution at $1 \mathrm{~km}$ (BESS) or 500m (MOD17A2 version 6). So for during the downscale, the reanalysis climate data need to resample, the 
spatial mismatch factor can not be neglected.

In this research we want to evaluate the difference between geostationary, in-situ measurement and reanalysis meteorology data. Then we analyzed the result of different spatial and temporal resolution input in GPP models and its estimation accuracy.

\section{METHODS AND DATA}

The US-NE2 site $(41.1649 \mathrm{~N}, 96.4701 \quad \mathrm{~W}$, http://fluxnet.fluxdata.org/ ) was used to be the study area in this research, where is the cropland in middle east of the USA. Study period was from 2010 to 2012.

GPP were modeled by MOD17 algorithm, was calculated like the following function:

$G P P=L U E \max \times f(T) \times g(V P D) \times F P A R \times S W a r d \times 0.45$

LUEmax in US-NE2 was set as $2.1 \mathrm{gC} / \mathrm{m} 2 /$ day in this study. SWard is the shortwave downward radiation obtained by satellite or meteorology data. The GOES-13 geostationary satellite digital number (DN) values based on remote sensing data and ERA-Interim reanalysis meteorology data were used to be the weather data input. The daily, 8-day, 16-day meteorology data input were the mean value for the flux tower based in-situ measurement data and reanalysis data. In order to evaluate the difference between satellite remote sensing data and ground based data, we also compared the geostationary visible band data onto the downward shortwave radiation data from flux tower and ERA reanalysis meteorology data. $\mathrm{f}(\mathrm{T})$ and $\mathrm{g}(\mathrm{VPD})$ were calculated by air temperature and water vapor pressure deficit (VPD) (Running et al, 2004) .

Landsat data is the high spatial resolution remote sensing data in this study and the MODIS product of $1 \mathrm{~km}$ represented for low resolution data. The Landsat based NDVI, MOD13 NDVI/EVI product were used to as vegetation growing condition information.

$$
F P A R=a \times V I+b
$$

where, VI represented for NDVI and EVI, $a$ and $b$ are the empirical coefficient from Xiao et al. (2005). Then we combined FPAR and meteorology data in LUE model (equation.1).

Finally, different frequency (daily, 8-day, 16-day), spatial resolution $(30 \mathrm{~m}, 1 \mathrm{~km})$, source data (in-situ measurement, reanalysis meteorology dataset) were input as the LUE model import for GPP estimation, so we get different temporal and spatial scale based GPP results. To validate multiple data input in LUE based GPP model, we used in-situ measurement carbon flux data as 'true value', then evaluated the GPP modeled results.

\section{RESULTS}

\subsection{Comparison of meteorology data and geostationary remote sensing data}

PAR was the most important factor of photosynthesis, determined the incident energy of photosynthesis process. GOES-13 provided hourly images of a certain observation angle from satellite. In band 1 of GOES-13 imager instrument, its DN value represented for shortwave radiation reflectance from top of the atmosphere. But as the geostationary remote sensing signal would affect by the zenith angle, it would hard to compare different period's DN value and in-situ measurement results without BRDF correction. So we compared GOES-13 imager band $1 \mathrm{DN}$ value and flux tower based in-situ measurement downward shortwave radiation in different time when the sun angle is almost the same in this section (Figure 1-3).

Figure.1-3a,b stated that it has negative correlation between shortwave radiation incident and GOES-13's reflectance signal: when incident shortwave radiation increases, the GOES-13 DN value decreases. Because GOES-13's high DN value represented for the observation area which had high percentage of cloud cover, the downward shortwave radiation would be lower at that time. Figure.2a showed that GOES-13 DN values had highest correlation $\left(\mathrm{R}^{2}=0.58\right)$ with in-situ measurement data at 12:00 during daytime, and ERA reanalysis meteorology data have similar result with GOES-13 data (Figure.2b). However, Figure.1a,b and Figure.3a,b illustrated the relevance between geostationary data with in-situ measurement data is lower in 9:00 and 15:00 $\left(\mathrm{R}^{2}<0.4\right)$. It showed similar relationship between reanalysis meteorology data and GOES-13 data.

On the other hand, the ERA reanalysis meteorology data, as a satellite based remote sensing product inverse from geostationary data, have high relationship of GOES-13 data in different time (Figure.1-3b). Figure.1-3c explained that ERA' downward shortwave radiation also has high correlation with in-situ measurement data in 9:00, 12:00, 15:00 (mean $\mathrm{R}^{2}=0.64$ ). So the ERA data represented the real condition of downward shortwave radiation in the land surface for the high frequency meteorology data input in GPP models.

However, in order to getting the land surface incident PAR from the geostationary data, it need to develop new algorithm to correct the value from satellite sensors (Hilker et al, 2008). 

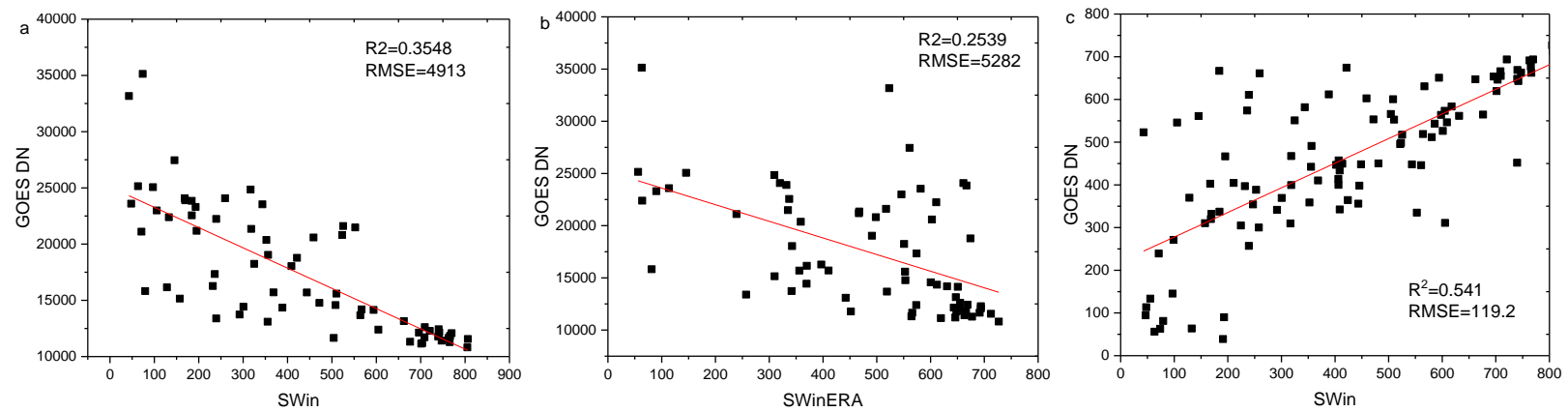

Figure.1 Comparison of satellite based DN values with in-situ measurement Shortwave radiation input in 9:00. GOES DN is the GOES-13 visible band's record. SWinERA is the ERA reanalysis downward shortwave radiation and SWin is the flux tower in-situ measurement downward shortwave radiation (in W/m2).
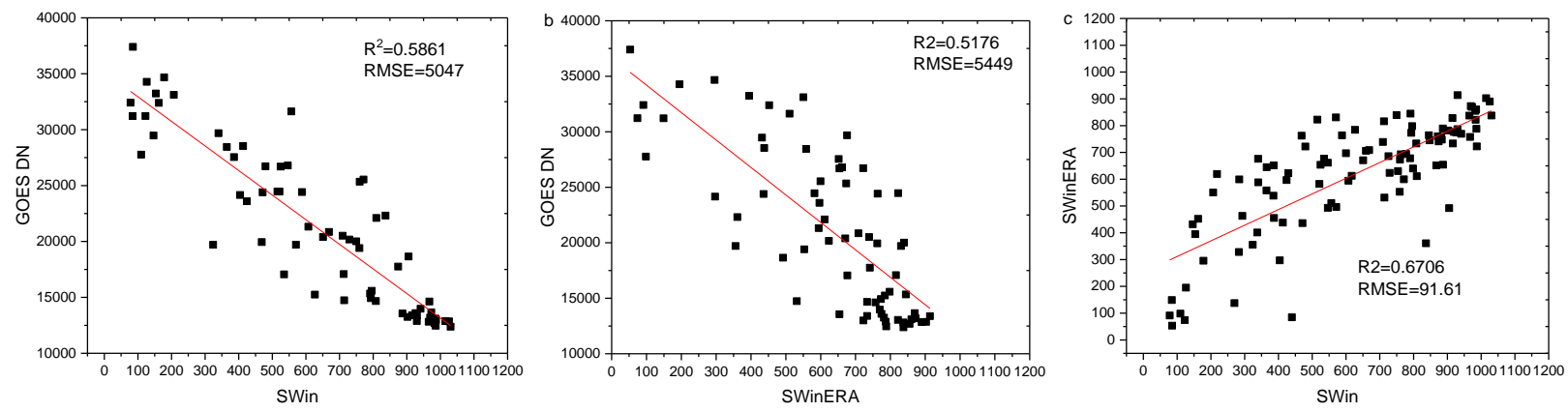

Figuer.2. Comparison of satellite based DN values with in-situ measurement Shortwave radiation input in 12:00. GOES DN is the GOES-13 visible band's record. SWinERA is the ERA reanalysis downward shortwave radiation and SWin is the flux tower in-situ measurement downward shortwave radiation (in $\mathrm{W} / \mathrm{m} 2$ ).
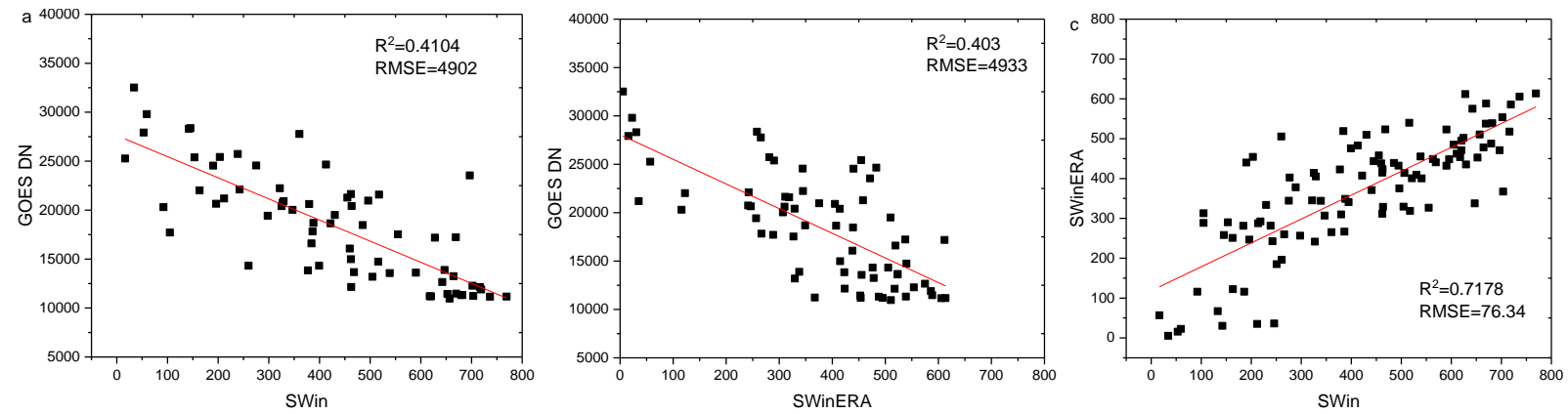

Figure.3. Comparison of satellite based DN values with in-situ measurement Shortwave radiation input in 15:00. GOES DN is the GOES-13 visible band's record. SWinERA is the ERA reanalysis downward shortwave radiation and SWin is the flux tower in-situ measurement downward shortwave radiation (in $\mathrm{W} / \mathrm{m} 2$ ).

\subsection{Comparison of different temporal and spatial scale GPP estimation results}

In section 3.1, we found that ERA data can reflect the land surface weather condition without in-situ measurement data to calibrate. In this section, daily, 8-day, 16-day ERA-Interim reanalysis data were used for meteorology data input for LUE model to estimate GPP in different spatial scale.

GPP estimation of different temporal scale showed different estimation accuracy. Although flux tower GPP showed similar trend with model based GPP, daily scale estimation RMSE would be higher than in 8-day and 16-day scale with MODIS NDVI and EVI input. GPP estimation accuracy ranged from daily $(\mathrm{R} 2=0.85, \quad \mathrm{RMSE}=3.05 \mathrm{gC} / \mathrm{m} 2 /$ day $), \quad 8$-day
(R2=0.75, RMSE=3.89 $\mathrm{gC} / \mathrm{m} 2 /$ day $), \quad 16$-day $\quad(\mathrm{R} 2=0.76$, $\mathrm{RMSE}=3.78 \mathrm{gC} / \mathrm{m} 2 /$ day) meteorology data input with Landsat NDVI as vegetation condition import.

GPP estimation of different spatial scale also showed different estimation accuracy. For example, table. 3 showed that in 16-day scale GPP estimation, model performance of Landsat $30-\mathrm{m}$ spatial scale NDVI resampled into $1-\mathrm{km}$ scale $(\mathrm{R} 2=0.76$, $\mathrm{RMSE}=3.8 \mathrm{gC} / \mathrm{m} 2 /$ day) is better than $1-\mathrm{km}$ MODIS NDVI input ( $\mathrm{R} 2=0.68, \mathrm{RMSE}=4.2 \mathrm{gC} / \mathrm{m} 2 /$ day). They also have similar estimation accuracy results from daily and 8-day temporal scale data input (Table.1,2). 1-km EVI and NDVI input based on MODIS data showed similar GPP estimation accuracy in different temporal scale.

Also, diverse source meteorology data input also lead to different result. Using ERA reanalysis data as input would have 
higher estimation accuracy than flux tower based data in different spatial and temporal scale's result. Table.1 illustrated that with daily scale data input, GPP estimation result based on
ERA meteorology data input has lower RMSE than with flux tower based meteorology data input.

Table.1 Comparison of remote sensing based GPP estimation with in-situ measurement carbon flux data in flux tower in daily temporal scale. Here the M-NDVI and M-EVI represented for GPP estimation results from the 1-km MODIS based vegetation index product input. L-NDVI represented GPP estimation results from the 30-m resolution LANDSAT NDVI resampled to the same region in 1-km MODIS data input. 1day-FLUX is using the in-situ measurement meteorology data as input, while 1day-ERA using reanalysis meteorology data as input data for GPP estimation (RMSE and MEANbias ${ }^{1}$ in $\mathrm{gC} / \mathrm{m} 2 /$ day).

\begin{tabular}{lcccccc}
\hline & \multicolumn{3}{c}{ 1day-FLUX } & \multicolumn{3}{c}{ 1day-ERA } \\
\hline & $\mathrm{R}^{2}$ & RMSE & MEANbias & $\mathrm{R}^{2}$ & RMSE & MEANbias \\
M-NDVI $\left(1 \mathrm{~km}^{2}\right)$ & 0.66 & 4.871 & 0.96 & 0.65 & 4.583 & 0.76 \\
M-EVI $\left(1 \mathrm{~km}^{2}\right)$ & 0.64 & 4.736 & 1.46 & 0.63 & 4.409 & 1.26 \\
L-NDVI $\left(\right.$ mean value in $\left.1 \mathrm{~km}^{2}\right)$ & $\mathbf{0 . 8 5}$ & $\mathbf{3 . 1 6 3}$ & $\mathbf{1 . 0 2}$ & $\mathbf{0 . 8 5}$ & $\mathbf{2 . 9 9 3}$ & $\mathbf{0 . 7 9}$ \\
\hline
\end{tabular}

Table.2 Comparison of remote sensing based GPP estimation with in-situ measurement carbon flux data in flux tower in 8-day temporal scale. Here the M-NDVI and M-EVI represented for GPP estimation results from the 1-km MODIS based vegetation index product input. L-NDVI represented GPP estimation results from the 30-m resolution LANDSAT NDVI resampled to the same region in 1-km MODIS data input. 8day-FLUX is using the in-situ measurement meteorology data as input, while 8day-ERA using reanalysis meteorology data as input data for GPP estimation(RMSE and MEANbias in $\mathrm{gC} / \mathrm{m} 2 / \mathrm{day}$ ).

\begin{tabular}{lcccccc}
\hline & \multicolumn{3}{c}{ 8day-FLUX } & \multicolumn{3}{c}{ 8day-ERA } \\
\hline & $\mathrm{R}^{2}$ & RMSE & MEANbias & $\mathrm{R}^{2}$ & RMSE & MEANbias \\
M-NDVI $\left(1 \mathrm{~km}^{2}\right)$ & 0.68 & 4.448 & 0.57 & 0.7 & 4.296 & 0.55 \\
M-EVI $\left(1 \mathrm{~km}^{2}\right)$ & 0.67 & 4.299 & 1.04 & 0.68 & 4.169 & 1.01 \\
L-NDVI (mean value in $\left.1 \mathrm{~km}^{2}\right)$ & 0.75 & 3.92 & 0.59 & 0.75 & 3.85 & 0.57 \\
\hline
\end{tabular}

Table.3 Comparison of remote sensing based GPP estimation with in-situ measurement carbon flux data in flux tower in 16-day temporal scale. Here the M-NDVI and M-EVI represented for GPP estimation results from the 1-km MODIS based vegetation index product input. L-NDVI represented GPP estimation results from the 30-m resolution LANDSAT NDVI resampled to the same region in 1-km MODIS data input. 16day-FLUX is using the in-situ measurement meteorology data as input, while 16day-ERA using reanalysis meteorology data as input data for GPP estimation (RMSE and MEANbias in $\mathrm{gC} / \mathrm{m} 2 / \mathrm{day}$ ).

\begin{tabular}{lcccccc}
\hline & \multicolumn{3}{c}{ 16day-FLUX } & \multicolumn{3}{c}{ 16day-ERA } \\
\hline & $\mathrm{R}^{2}$ & RMSE & MEANbias & $\mathrm{R}^{2}$ & RMSE & MEANbias \\
M-NDVI $\left(1 \mathrm{~km}^{2}\right)$ & 0.7 & 4.357 & 0.57 & 0.71 & 4.228 & 0.55 \\
M-EVI $\left(1 \mathrm{~km}^{2}\right)$ & 0.68 & 4.211 & 1.04 & 0.69 & 4.107 & 1.01 \\
L-NDVI $\left(\right.$ mean value in $\left.1 \mathrm{~km}^{2}\right)$ & 0.76 & 3.8 & 0.6 & 0.76 & 3.767 & 0.58 \\
\hline
\end{tabular}

1 MEANbias is mean modeled GPP minus mean flux tower measurement GPP. 


\section{DICUSSION AND CONCLUSION}

In this study, we estimated GPP with high spatial resolution and high temporal frequency meteorology data in cropland area. This study evaluated GPP estimation accuracy influenced by three major aspects of data input, including: i) different spatial scale remote sensing vegetation index input like 30-m resolution Landsat NDVI, 1-km resolution MODIS NDVI; ii) different frequency meteorology data input in GPP model like daily, 8-day, 16-day; iii) different source of data input for GPP estimation like flux tower based data input and ERA reanalysis data input. Results elucidated that GPP estimation with high spatial resolution remote sensing NDVI (Landsat based 30-m NDVI) and daily ERA reanalysis meteorology data input has the highest correlation with in-situ measurement GPP data in cropland area (R2=0.85, RMSE $=2.99$ ).

In the spatial scale, estimating GPP with high spatial resolution data like 30-m Landsat NDVI, has the higher estimating accuracy than using 1-km MODIS NDVI as input. Because high spatial resolution data have higher representative of land surface vegetation growing condition than low resolution data. New generation satellite based remote sensing data provided different spatial resolution of vegetation information like GaoFen in 10-m (Zhao et al, 2018), Sentinel-2a in 16-m (Battude et al,2016), VIIRS in 500-m (Justice et al,2010), all of these can be applied to remote sensing GPP estimation models. So the further remote sensing based GPP products can use the high spatial resolution information as import.

In the temporal scale, evaluating GPP with daily meteorology data input and Landsat based high spatial resolution vegetation information has highest accuracy. Because high frequency weather data input changes the stress for vegetation growing then tracking vegetation production variation. However, only geostationary satellites and meteorology reanalysis dataset provide high frequency land surface weather condition data (Bessho et al,2016). In section 3.1, geostationary images not have very high estimation correlation between in-situ measurement meteorology data. Some research found that the atmosphere and BRDF correction of geostationary need to have further improvement so it has high frequency data as the GPP model input (Bessho et al,2016 ,Hilker et al, 2008).

Remote sensing based GPP estimation accuracy influenced by model parameters input: vegetation index input like NDVI, EVI; in-situ measurement or reanalysis meteorology input. In this study, we found that estimating GPP with NDVI and ERA based reanalysis data have the best performance in MOD17 GPP model. But it needs to have more research on other models like VPM (Xiao et al,2005; Dong et al, 2016), BESS(Jiang et al, 2016) etc.

Estimating GPP with high spatial and temporal remote sensing based data can improve its accuracy, which would be a potential way to improve crop production in large scale cropland region.

\section{REFERENCES}

Monteith, J. L. (1972). Solar radiation and productivity in tropical ecosystems. Journal of Applied Ecology, 9(3), 747-766. Ryu, Y., Jiang, C., Kobayashi, H., \& Detto, M. (2017). Modis-derived global land products of shortwave radiation and diffuse and total photosynthetically active radiation at $5 \mathrm{~km}$ resolution from 2000. Remote Sensing of Environment.

Kalfas, J. L., Xiao, X. M., Vanegas, D. X., Verma, S. B., \& Suyker, A. E. (2011). Modeling gross primary production of irrigated and rain-fed maize using modis imagery and co2 flux tower data. Agricultural \& Forest Meteorology, 151(12), $1514-1528$

Suyker, A. E., \& Verma, S. B. (2012). Gross primary production and ecosystem respiration of irrigated and rainfed maize-soybean cropping systems over 8 years. Agricultural \& Forest Meteorology, 165(6), 12-24.

Maselli, F., Papale, D., Puletti, N., Chirici, G., \& Corona, P. (2009). Combining remote sensing and ancillary data to monitor the gross productivity of water-limited forest ecosystems. Remote Sensing of Environment, 113(3), 657-667. Nguy-Robertson, A., Suyker, A., \& Xiao, X. (2015). Modeling gross primary production of maize and soybean croplands using light quality, temperature, water stress, and phenology. Agricultural \& Forest Meteorology, 213, 160-172.

Field, C. B., Jackson, R. B., \& Mooney, H. A. (1995). Stomatal responses to increased co 2 : implications from the plant to the global scale. Plant Cell \& Environment, 18(10), 1214-1225.

STEVEN W. RUNNING, RAMAKRISHNA R. NEMANI, FAITH ANN HEINSCH, MAOSHENG ZHAO, MATT REEVES, \& HIROFUMI HASHIMOTO. (2004). A continuous satellite-derived measure of global terrestrial primary production. BioScience, 54(6), 547-560.

Xiao, X., Hollinger, D., Aber, J., Goltz, M., Davidson, E. A., \& Zhang, Q., et al. (2004). Satellite-based modeling of gross primary production in an evergreen needleleaf forest. Remote Sensing of Environment, 89(4), 519-534.

Pathmathevan Mahadevan, Steven C. Wofsy, Daniel M. Matross, Xiangming Xiao, Allison L. Dunn, \& John C. Lin, et al. (2008). A satellite - based biosphere parameterization for net ecosystem co2 exchange: vegetation photosynthesis and respiration model (vprm). Global Biogeochemical Cycles, 22(2), 521-539.

Wenping Yuan, Shuguang Liu, Guangsheng Zhou, Guoyi Zhou, Larry L. Tieszen, \& Dennis Baldocchi, et al. (2007). Deriving a light use efficiency model from eddy covariance flux data for predicting daily gross primary production across biomes. Agricultural and Forest Meteorology, 143(3-4), 189-207.

Zhao, J., Li, J., Liu, Q., Wang, H., Chen, C., \& Xu, B., et al. (2018). Comparative analysis of chinese hj-1 ccd, gf- $1 \mathrm{wfv}$ and zy-3 mux sensor data for leaf area index estimations for maize. Remote Sensing, 10(1), 68.

Battude, M., Bitar, A. A., Morin, D., Cros, J., Huc, M., \& Sicre, C. M., et al. (2016). Estimating maize biomass and yield over large areas using high spatial and temporal resolution sentinel-2 like remote sensing data. Remote Sensing of Environment, 184, 668-681.

Justice, C. O., Vermote, E., Privette, J., \& Sei, A. (2010). The evolution of u.s. moderate resolution optical land remote sensing from avhrr to viirs. , 11, 781-806.

Bessho, K., Date, K., Hayashi, M., Ikeda, A., Imai, T., \& Inoue, H., et al. (2016). An introduction to himawari-8/9- japan's new-generation geostationary meteorological satellites. Journal of the Meteorological Society of Japan.ser.ii, 94.

Xiao, X., Boles, S., Liu, J., Zhuang, D., Frolking, S., \& Li, C., 
et al. (2005). Mapping paddy rice agriculture in southern china using multi-temporal modis images. Remote Sensing of Environment, 95(4), 480-492.

Dong, J., Xiao, X., Menarguez, M. A., Zhang, G., Qin, Y., \& Thau, D., et al. (2016). Mapping paddy rice planting area in northeastern asia with landsat 8 images, phenology-based algorithm and google earth engine. Remote Sensing of Environment, 185, 142.

Jiang, C., \& Ryu, Y. (2016). Multi-scale evaluation of global gross primary productivity and evapotranspiration products derived from breathing earth system simulator (bess). Remote Sensing of Environment, 186, 528-547.

Hilker, T., Coops, N. C., Hall, F. G., Black, T. A., Wulder, M. A., \& Nesic, Z., et al. (2008). Separating physiologically and directionally induced changes in pri using brdf models. Remote Sensing of Environment, 112(6), 2777-2788.

Running, S. W., Nemani, R. R., Heinsch FAZhao, M. S.,

Reeves, M., \& Hashimoto, H. (2004). A continuous satellite-derived measure of global terrestrial primary production. Bioscience, 54(6), 547-560. 